\title{
Spatial Reuse for Practical Scenarios: Iterative Power Adjustment from Distributed Contour Estimation and Propagation
}

\author{
Sofie Pollin ${ }^{1,3}$ Bart Adams ${ }^{2,4}$ Ahmad Bahai ${ }^{1,2}$ \\ ${ }^{1}$ University of California Berkeley E-mail : \{pollins\}@eecs.berkeley.edu \\ ${ }^{2}$ Stanford University $\quad{ }^{3}$ Interuniversity Micro-Electronics Center ${ }^{4}$ Katholieke Universiteit Leuven
}

\begin{abstract}
The number of wireless networks that coexist in space is increasing steeply. To allow coexistence while avoiding interference, it becomes important to properly characterize the propagation contour where the received power of wireless transmitters reaches a certain threshold. Detailed channel modeling taking into account the specificities of typical urban scenarios is however a very complex task. Thanks to the widespread use of wireless access technology, it becomes feasible to use network nodes to estimate and communicate these propagation contours. In this paper, we propose a lightweight practical scheme for local contour estimation of a given transmitter. The local estimate is efficiently propagated to all other secondary transmitters that can then meet interference constraints optimally, i.e., without having to consider large safety margins that limit spatial reuse gains. This optimality is obtained through iterative power control based on true propagation contour distances and local pathloss estimates. The overhead of the estimation and communication phases is simulated to be close to linear in the number of nodes, so that the solution scales well. The scheme can be used for optimal power control in practical wireless networks, or for the deployment of secondary networks in areas with primary transmitters that should be protected.
\end{abstract}

\section{INTRODUCTION}

Due to significant improvements in semiconductor technology, the use of wireless techniques in every electronic device has become possible. As a result, more and more applications rely on wireless technology and more spectrum is required to accommodate these applications. Techniques are being proposed to make better use of existing spectrum resources in time, space and frequency. Given the fact that wireless communication is inherently dynamic, efficiency can be obtained through a more adaptive spectrum use. How to achieve this given practical constraints is a very important research challenge today [1].

Controlling or adapting spectrum use in space is typically achieved using Transmit Power Control (TPC). Many techniques have been proposed to achieve optimal power control through central coordination or in a distributed setting [2]. Each of these techniques however requires a detailed knowledge of the propagation conditions of two conflicting transmitters towards their intended receivers. When it is not possible to rely on such channel state feedback from the receivers, this information is typically obtained through sensing for transmitters. To detect potentially shadowed existing transmitters, the sensitivity requirements of secondary transmitters



Fig. 1. Spatial reuse in wireless networks requires high sensitivity receivers and moreover never achieves optimal adaptation to the real propagation conditions, since safety margins are needed to avoid interference to the potential receivers with unknown channels. We want to achieve optimal spatial reuse (i.e., without safety margins that limit the gain), while relaxing the receiver sensitivity constraints.

are very high [3]. Even when the required sensitivity can be achieved, the allowed power for the secondary transmitter is typically much lower than the power that could actually be tolerated, since large safety margins are required to account for unpredictable shadowing. Indeed, as illustrated in Fig. 1, the channel sensed between the primary and secondary transmitter only reflects the channel characteristics along this specific path and safety margins need to be introduced to avoid interference along other paths towards the receivers.

To improve spatial planning of networks, we propose a distributed and practical algorithm that

(a) relaxes the sensitivity requirements and hence allows for less expensive hardware and more practical deployments,

(b) achieves optimal power allocation as a function of actual and arbitrary propagation conditions.

Moreover, we will show that our algorithm scales well and is robust against measurement noise.

We develop our model based on measurements of the propagation characteristics of outdoor IEEE 802.11 networks where one Access Point (AP) is considered to be a primary AP and a second AP should avoid interfering with it. We also illustrate the performance on a simulated network with similar real-world noise characteristics. However, our technique is not limited to this context and will also benefit spatial reuse in other networks that exhibit highly irregular and difficult to predict propagation characteristics. For example, in the context of IEEE 802.22 Wireless Regional Area Networks (WRAN), there is a desire to re-use the TV spectrum in areas where certain TV bands are not used [4]. The traditional approach of GPS assisted distance-based power control coupled with 


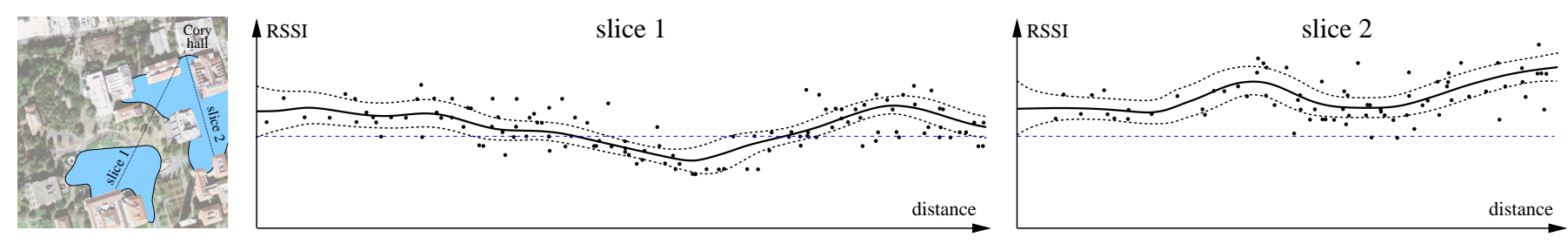

Fig. 2. RSSI measurements for an outdoor 802.11 antenna located on top of Cory Hall at UC Berkeley. The left image shows the resulting contours for a given RSSI threshold, as we will compute in Sec. IV. The two right images show measurement points and denoised RSSI signals with $95 \%$ confidence intervals for two different slices. The horizontal dashed line corresponds to the RSSI contour threshold on the left image. Note the high noise levels, the absence of a clear trend and the very different results along the two directions.

high sensitivity requirements of secondary transmitters results in poor spatial reuse because the power determined from the distance will have to reflect worst-case propagation conditions. Our technique could be used to communicate actual propagation contours of TV transmitters and improve on the overly conservative power estimates.

We do rely on the availability of nodal positioning information which could be obtained through GPS measurements or a localization algorithm. We also assume that for the sake of determining the optimal power, a limited amount of interference from control messages is tolerated. Standards dealing with coexistence specify the time during which such control communication is allowed [4].

The remainder of this paper is organized as follows. First, in Sec. II, we give an overview of the outdoor measurements, illustrating many of the problems arising when estimating propagation characteristics. Next, in Sec. III, we give a general overview of our solution and how it differs from existing ones, motivated by aforementioned problems. Sec. IV, V and VI detail the main steps of our algorithm (i.e., contour estimation, contour propagation and power adaptation). We conclude our paper with a discussion of results in Sec. VII.

\section{OUTDOOR 802.11 MEASUREMENTS}

When designing the contour estimation and propagation method, it is important to make the correct assumptions. Typically, wireless propagation is characterized by means of a distance-dependent pathloss or trend. On top of this trend, shadowing losses are added that are assumed to vary slowly. Finally, fast fading due to multipath effects introduces variations at very small timescales. We verify the impact of each of those components through measurements. This motivates our general contour estimation method.

\section{A. Measurement Setup}

Measurements were obtained from the outdoor 802.11 network at the UC Berkeley campus. A highly accurate GPS receiver (with an error below $1 \mathrm{~m}$ ) provides location information. Since this is very accurate compared to the received power measurements, we will neglect positioning errors. At each location, we obtain the Received Signal Strength Indication (RSSI) which is a measure of received power for each beacon received from the various 802.11 Access Points (AP) on campus.

A reconstructed contour using our algorithm for one of the APs is shown on the left of Fig. 2. The two graphs in the middle and the right of the figure show pathloss measurements along the slices indicated on the left overview image. Each of the continuous pathloss curves in Fig. 2 is obtained by computing for each point along the horizontal axis a local moving average (details are discussed in Sec. IV). Along with the moving average, we compute the variance, and hence $95 \%$ confidence intervals (assuming the error is normally distributed), as indicated on the figures.

\section{B. Observations from Measurements}

From these measurements, we make following observations.

1) No Clear Trend: As can be seen in the slices drawn from the measured signal strength in Fig. 2, it is not possible to find a clear trend in the pathloss as function of distance. Reception can degrade significantly behind a building, and then improve again at larger distances.

2) Anisotropic due to Shadowing: As shown in Fig. 2, the propagation trend looks very different along the two paths. We hence conclude that it is not possible to fit a single trend to the measurements and we will propose a technique to fit local trends, not relying on the assumption that a single model fits the entire propagation surface properly.

3) Noisy Measurements due to Fast Fading: The RSSI sample points in Fig. 2 appear very noisy. We will propose a technique to locally smooth out the noise and obtain the solid curve along with associated confidence intervals.

We will propose a local channel estimation strategy to combat both (1) and (3) and present a technique to communicate the local estimate through the network and achieve a power control considering not only distance, but also direction as motivated by conclusion (2).

\section{Algorithm Overview AND Design Decisions}

The problem we want to solve can be stated as follows. For each potential secondary transmitter, we want to compute its allowable transmission power, so that a maximum number of receiving nodes can be reached without interfering with (potentially multiple) primary transmitters. In the following we will show that this amounts to minimizing received power contour-to-contour distances.

On a high level our algorithm works as follows. First, nodes which sense the primary transmitter compute a robust estimate of the local RSSI. Next, the shortest distance to the interior nodes, i.e., nodes within the propagation contour or with RSSI above a given threshold, is propagated through the network. This distance can be used by a secondary transmitter to conservatively estimate an initial transmission power. Next, the secondary transmitter iteratively estimates the minimal 
distance between the primary signal threshold contour and its own secondary interference threshold contour and uses this contour-to-contour distance along with a local estimate of the pathloss model to adapt its transmission power to improve the spatial reuse gain.

We now discuss the design decisions for these three steps which are based on the aforementioned observations in realworld wireless networks. The algorithmic details will be discussed in Sec. IV, V and VI.

\section{A. Local Channel Estimation}

Adapting the spatial resource allocations at the time-scale of fast fading is difficult to achieve in practical scenarios when the time to communicate a change is often much longer than the time during which the change is in effect. The importance of averaging out those fast-fading effects for power control was already noted in [5], where the number of samples needed to achieve a target confidence interval of the slow pathloss estimate is computed for a theoretical fading model.

Since we are interested in using power control for spatial resource optimization, it is also required to average out variations over small areas. Large-scale distance-related pathloss and shadowing due to large objects result in large effects that should be considered in the spatial planning. However, fast fading due to multipath effects gives rise to very large variations over distances in the order of a single wavelength. Similar to the motivation above where fast fading effects make it impossible to adapt timely, it is clear that spatial planning using power control cannot take advantage of channel variations over very small areas.

In addition to averaging over time, we therefore propose in Sec. IV a spatial Moving Least Squares (MLS) algorithm to obtain a local estimate RSSI of the received power measure RSSI. MLS is a powerful regression technique to smooth out noise, while obtaining the best local fit of the propagation trend. As mentioned also in [6], and verified by our measurements, it is impossible to rely on a known propagation trend, so fitting this trend adaptively and locally without relying on any false model proves to be a very robust approach.

\section{B. Distance-to-Contour Flooding}

Propagating or broadcasting information in a network is typically achieved through flooding. In [7] it is shown that in ad hoc wireless networks, the more important cost factor for flooding is the number of packet forwardings and it is important to prune away unnecessary transmissions.

For reporting a measured value to a sink, many schemes have been proposed in the literature in the context of sensor networks [8]. An interesting approach is found in [9], where the authors study the problem of delivering messages from any sensor to an interested client user along the minimum-cost path in a large sensor network. For a single client, they can establish the optimal cost field with $N$ messages, through the use of a clever backoff approach. This approach establishes a tree from any sensor to a client. We will propose a similar algorithm which propagates pathloss information from any receiver to any potential transmitter, hence conceptually resulting in a forest of trees. The resulting flooding method as detailed in Sec. V requires a number of packet forwardings close to the number of nodes $N$.

\section{Iterative Power Control}

When each secondary transmitter knows its distance to the first transmitter's contour, it can compute its maximal power as function of the interference margins and a worst-case pathloss model as function of distance. We note that from a secondary transmitter's viewpoint, a worst-case pathloss model is an optimistic one that assumes that the power of the secondary transmitter decays slowly, resulting in maximal interference. Consequently, the computed power level will be much lower than what could be tolerated in reality.

Using the approach of contour estimation and distance flooding, we can however iteratively adapt the distance between the two contours. While estimating the contour of the secondary transmitter, we will also estimate a more realistic pathloss model which in turn will be used to improve the power estimate. Key here is to estimate the pathloss in the direction of the point closest to the first transmitter's contour. Since the pathloss model can vary significantly as a function of the exact path followed, this direction gives the best information for the targeted power control. As will be shown in Sec. VI, this iterative power adjustment algorithm will converge in only a few steps and will require an order of $N$ communications per secondary transmitter.

\section{Local Channel Estimation}

In this section, we will detail how a node can locally smooth the fast RSSI variations, without relying on a global trend, which allows robust inside/outside classification with respect to the threshold contour.

Given a node $N_{i}$ at location $\mathbf{x}_{i}$, with received (noisy) power measure $\mathrm{RSSI}_{i}$, we wish to find the noise-reduced power $\operatorname{RSSI}_{i}$. Given a complete polynomial basis $\mathbf{p}(\mathbf{x})=$ $\left[\begin{array}{llll}1 & \mathbf{x} & \ldots & \mathbf{x}^{n}\end{array}\right]^{T}$, we try to find the coefficient vector $\hat{\mathbf{a}}_{i}$ which minimizes following weighted least squares objective function:

$$
\hat{\mathbf{a}}_{i}=\arg \min _{\mathbf{a}} \sum_{j} w_{i j}\left(\mathbf{a}^{T} \mathbf{p}\left(\mathbf{x}_{j}\right)-\mathrm{RSSI}_{j}\right)^{2},
$$

where the summation is over $N_{i}$ 's neighboring nodes $N_{j}$ (with positions $\mathbf{x}_{j}$ ). Note that $N_{i}$ is contained in its own neighborhood. For example, the complete polynomial basis of order $n=1$ in two dimensions is $\mathbf{p}(\mathbf{x})=\left[\begin{array}{lll}1 & x & y\end{array}\right]^{T}$ and the coefficient vector $\mathbf{a}=\left[\begin{array}{lll}a_{0} & a_{1} & a_{2}\end{array}\right]^{T}$ consists of three unknowns. We will give results for moving least squares approximations of order $n=0,1$ and 2 .

The filtered RSSI at the node $N_{i}$ is then given by:

$$
\mathrm{RSSI}_{i}=\hat{\mathbf{a}}_{i}^{T} \mathbf{p}\left(\mathbf{x}_{i}\right) \text {. }
$$

Locality is obtained by weighting the neighboring nodes' contributions using the distance-based weight function $w_{i j}$ with local support $h$ :

$$
w_{i j}= \begin{cases}\left(1-r_{i j}^{2}\right)^{3} & \text { if } r_{i j} \leq 1 \\ 0 & \text { otherwise }\end{cases}
$$


where $r_{i j}=\left(\left\|\mathbf{x}_{i}-\mathbf{x}_{j}\right\|\right) / h$.

So, although in practice we only fit a bi-variate polynomial at each nodal position, the resulting RSSI is defined everywhere and will be $C^{2}$-continuous thanks to the above defined smoothly vanishing weight function. This weight function decays fast enough to establish a true local fit [10]. Note that each node computes its smoothed $\mathrm{RSSI}_{i}$ estimate independently and only requires local RSSI and GPS localization information to do so, which can be obtained by a single communication step with its neighboring nodes if $h$ is equal or smaller than the communication range of the secondary network users. In the remainder, we will assume that $h$ is the communication range. This step hence requires $N$ communications in the network.

It is easy to see that for order $n=0$, the minimizer of Eq. 1 equals the weighted average measured RSSI:

$$
\operatorname{RSSI}_{i}=\frac{\sum_{j} w_{i j} \mathrm{RSSI}_{j}}{\sum_{j} w_{i j}}
$$

and thus the moving least squares approximation coincides with the well-known moving average approximation method.

Intuitively, when the main goal of the smoothing operation is to average out spatial variations, the moving average will result in adequate results. Alternatively, in case larger neighborhoods are used and more measurement points are collected, it might make sense to fit a local trend (e.g., linear or quadratic). Such a trend will reduce the smoothing effect on the one hand, but will improve accuracy on the other hand since there are more degrees of freedom for the parameters. We illustrate this in Fig. 3 (left) using a simulated scenario (see also Sec. VII) where we have a known contour and systematically increase the noise of the RSSI measurements. As can be seen on the figure, when less smoothing is required (less noise power), a quadratic MLS approximates the propagation surface best. However, with increasing noise power more smoothing is required and linear and constant MLS start to outperform.

Choosing the order of approximation as function of the neighborhood size is a delicate trade-off. The choice of local support $h$ as used in Eq. 3 influences the approximation quality. As shown in Fig. 3 (right), an optimal kernel width can be found for all approximation orders and the optimal width typically increases with approximation order.

For the real-world RSSI measurements we observed a noise power of $\sigma_{N}^{2}=4$. At this noise level, quadratic MLS with kernel width or communication range of $95 \mathrm{~m}$ proved to be the best (cf. Fig. 3 (right)) and we used these settings for the simulation results provided in the following sections.

\section{Distance-to-Contour Flooding}

A node is now classified as interior with respect to the threshold contour if $\mathrm{RSSI}_{i} \geq \mathrm{RSSI}^{\text {contour }}$. By determining the distance of each node to the closest interior nodes, it is possible to establish an estimate of the shortest distance to the contour. From this distance we can then iteratively approximate the transmit power in Sec. VI. We will use a fast marching algorithm which bears similarities to Dijkstra's shortest path algorithm. However, instead of computing a
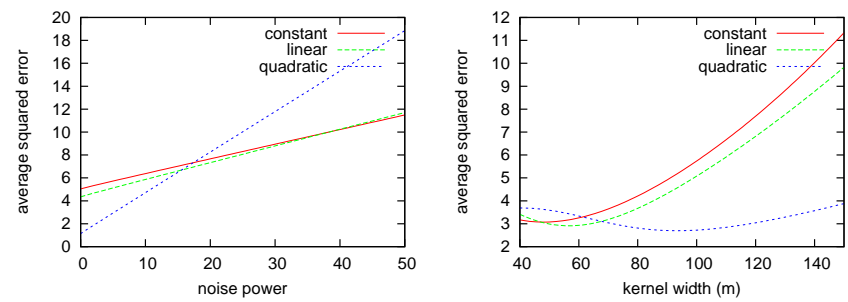

Fig. 3. Left: Average squared error of the moving least squares approximation (with fixed support radius $h$ ) for increasing noise power illustrated for order $n=0,1,2$. At low noise levels, quadratic MLS is superior, while with increasing noise levels, linear and then constant MLS have better performance. Right: Average squared error of the moving least squares approximation (with fixed noise power of 4) for varying kernel widths shown for order $n=$ $0,1,2$. Clearly, an optimal communication range $h$ can be found for each approximation order.

multihop distance, we will effectively compute a straight line distance to the contour, which is possible since we have GPS coordinates. First, we will discuss the centralized algorithm based on a priority queue. Next, we show how it can be made fully distributed.

\section{A. Centralized Distance-to-Contour Computation}

During the execution of the algorithm each node $N_{i}$ stores its distance $d_{i}$ to the closest interior node, or footpoint $\mathbf{f}_{i}$, inside the contour. The algorithm starts by setting $d_{i} \leftarrow 0$ and $\mathbf{f}_{i} \leftarrow \mathbf{x}_{i}$ for all interior nodes. The distance for all other nodes is set to infinity $\left(d_{i} \leftarrow \infty\right)$. The nodes with $d_{i}=0$ are added to a priority queue, which is sorted in increasing Distance-to-Contour order.

The algorithm proceeds iteratively by taking the first node $N_{i}$ from the queue (i.e., the one with the smallest $d_{i}$ ), and by updating its neighboring nodes $N_{j}$ (those within a communication distance $h$ to $N_{i}$ ). The footpoint $\mathbf{f}_{i}$ stored with $N_{i}$ is used for this. If $\left\|\mathbf{f}_{i}-\mathbf{x}_{j}\right\|<d_{j}$, i.e., if $N_{j}$ is closer to $N_{i}$ 's footpoint than it is to its previously computed own footpoint, we update $N_{j}$ 's distance and footpoint information $\left(d_{j} \leftarrow\left\|\mathbf{f}_{i}-\mathbf{x}_{j}\right\|\right.$ and $\left.\mathbf{f}_{j} \leftarrow \mathbf{f}_{i}\right)$ and add $N_{j}$ to the priority queue. This process is repeated until the queue becomes empty.

Due to the use of a priority queue this algorithm requires a central base station. However, we can approximate this algorithm and make it fully distributed by cleverly modifying the medium access protocol as discussed below.

\section{B. Distributed Distance-to-Contour Flooding}

When implementing the fast marching in a network, it is required that each node updates its neighbors by sending its footpoint information. We note that nodes that are inside the contour are footpoint to themselves, and hence have perfect information at the start of the algorithm. Inspired by the optimal centralized implementation of the algorithm, we note that it is optimal to have nodes with a smaller distance to the contour update their neighbors first. In practical networks, we can achieve this by giving those nodes a higher probability $p_{\text {send }}$ to gain access to the channel. While nodes are waiting to access the channel, they can accumulate neighbor update events and only propagate the best footpoint found so far. This probability $p_{\text {send }}$ is typically a function of a backoff 




Fig. 4. Black nodes (such as $\mathbf{f}_{1}$ and $\mathbf{f}_{2}$ ) correspond to interior nodes (i.e., nodes inside the contour). The straight lines trace the regions which are closest to a certain interior node (i.e., the Voronoi regions). Our new proposed backoff scheme ensures that nodes (such as $A$ and $B$ ) which are closer to the interior contour nodes are updated first before they propagate this contour distance information to the other nodes. This propagation can be performed with little more than $N$ transmissions, with $N$ the total number of nodes.

timer in practical protocols such as 802.11 , or alternatively, it is a function of the scheduling algorithm carried out at the AP.

In our simulation, we opted for a random backoff timer that determines when nodes update their neighbors. Assume that $\mathrm{CW}$ is the initial backoff window resulting in a negligible number of packet collisions. Since distance-to-contour information is spreading away from the contour, we want to make sure that nodes only send when all nodes in their neighborhood that are closer to the contour have sent first.

As illustrated in Fig. 4, we can be sure that all black footpoints $\mathbf{f}_{i}$ with backoff in the range $[0 \ldots C W$ [ will have sent after time $C W$, if we neglect collisions requiring retransmissions for the ease of reasoning. As a result, a node $A$ that receives an update from a footpoint $\mathbf{f}_{1}$ (with backoff $B_{f_{1}}$ in range $\left[0 \ldots C W[)\right.$ will have to wait an additional $C W-B_{f_{1}}$ after reception of $\mathbf{f}_{1}$ 's update. This can be achieved if $A$ selects a backoff in the range $\left[\left(C W-B_{f_{1}}\right) \ldots\left(C W-B_{f_{1}}\right)+C W[\right.$. Clearly, as indicated in Fig. 4, the updating speed of the network will be approximately ${ }^{1}$ the one-hop communication range $h$, each $C W$ timeslots.

We note however that the distributed implementation of the scheme can potentially result in a node spreading information before it obtained its closest footpoint. As a result, such a node will have to update its neighbors more than once. This is illustrated for node $C$ in Fig. 4. Node $C$ only receives information of node $B$ before time $2 C W$, and hence thinks its closest point on the contour is $\mathbf{f}_{2}$. Following the algorithm, $C$ will have chosen a random backoff in the range $\left[\left(C W-B_{B}\right) \ldots\left(C W-B_{B}\right)+C W[\right.$ to start updating its neighbors. If node $C$ does not receive correct information before that random time, wrong information will be propagated and node $C$ will have to update its neighbors a second time once information about the correct footpoint $\mathbf{f}_{1}$ is obtained. In practice, with this scheme, we however experienced a number of update events that was very close to the number of nodes (neglecting collisions) as also illustrated in Table I. The contour spreading as function of time is illustrated in Fig. 5 for a simulated scenario (724 transmission steps were used for a total of $N=710$ nodes for this example). We note that the

\footnotetext{
${ }^{1}$ Due to sampling irregularities, as illustrated in Fig. 4 also, the contours do not spread exactly over a distance $h$ each step.
}
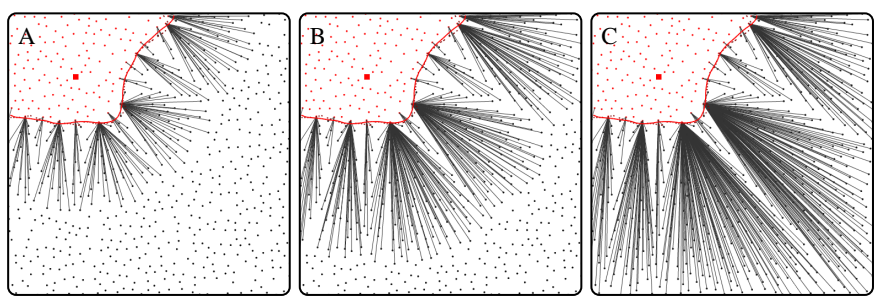

Fig. 5. Distributed distance-to-contour flooding shown at three intermediate timesteps during the algorithm for a simulated scenario.

algorithm will converge for arbitrary complex contour shapes, such as the one shown in Fig. 2 which consists of multiple parts.

\section{Iterative Power Control}

Given the learned shortest distance to the target contour, the secondary user decides conservatively on its transmission power. This initial transmission power is typically suboptimal and will now be improved by the following iterative procedure. Using the contour estimation method proposed in Sec. IV, nodes classify themselves as interior or exterior with respect to the secondary sender's target interference power contour. If no node inside this contour is within a target distance of the first transmitter's contour, the secondary transmitter should increase its power. The amount of power increase will be robustly determined by locally estimating the pathloss parameters as will be discussed below. Alternatively, in case the contours overlap, the opportunistic sender's power will have to be decreased. The number of messages needed to inform the secondary transmitter will be the number of interior nodes $N_{\text {interior }}$, which is smaller than or equal to $N^{2}$. This power adaptation is iterated until the distance between the contours is smaller than the one-hop communication distance $h$. We now discuss both cases (i.e., power increase and decrease).

\section{A. Increasing Power}

While estimating the secondary sender's propagation contour, interior nodes also robustly approximate the local pathloss parameters $\hat{\alpha}_{i}$ and $\hat{\beta}_{i}$ by minimizing following weighted least squares objective function:

$$
\left\{\hat{\alpha}_{i}, \hat{\beta}_{i}\right\}=\arg \min _{\alpha, \beta} \sum_{j} w_{i j}\left(\alpha 10 \log _{10}\left(r_{j}\right)+\beta-\mathrm{RSSI}_{j}\right)^{2},
$$

where $r_{j}=\left\|\mathbf{x}_{j}-\mathbf{x}_{s 2}\right\|$ is the distance from node $N_{j}$ to the secondary sender and $\alpha$ is the pathloss exponent and $\beta$ represents system losses. The summation is performed over the nodes $N_{j}$ in the local neighborhood around $N_{i}$, and the weighting is distance-based as given by Eq. 3. Due to this locality, each interior node $N_{i}$ will find a different estimate of those parameters. However, as we mentioned earlier in Fig. 2, it is indeed the case that pathloss can vary significantly as function of the exact direction. In the power control problem, it will be most useful to use the pathloss estimate of the node that will be closest to the first transmitter's contour.

\footnotetext{
${ }^{2}$ Here, we assume that each interior node can reach the secondary transmitter in a single hop, which is the case in most communication systems that are duplex.
} 
Interior nodes $N_{i}$ now send a message to the sender which includes following information: Estimated pathloss parameters $\hat{\alpha}_{i}$ and $\hat{\beta}_{i}$, footpoint $\mathbf{f}_{i}$ on the primary sender's contour and distance to this footpoint $d_{i}=\left\|\mathbf{x}_{i}-\mathbf{f}_{i}\right\|$. The secondary sender retains from all these messages the one with minimal $d_{i}$. The corresponding node $N_{i}$ is the interior node (with respect to the secondary sender's contour) which is closest to the primary contour and hence considered to give the best estimate of $\alpha$ and $\beta$. The distance $d=\left\|\mathbf{x}_{s 2}-\mathbf{f}_{i}\right\|$ from the secondary sender $\mathbf{x}_{s 2}$ to the footpoint $\mathbf{f}_{i}$ is used together with the estimated pathloss parameters to estimate an improved transmission power:

$$
P=P_{\text {interference }}+\hat{\alpha}_{i} 10 \log _{10}(d)+\hat{\beta}_{i},
$$

where $P_{\text {interference }}$ denotes the maximal interference that can still be tolerated at the established contour of the first sender.

This process of power adaptation based on the estimated pathloss parameters at the minimal contour-to-contour node can be iterated to further improve the secondary transmission power estimation. As can be seen in Fig. 6, this requires only a few iteration steps. In each step, one contour estimation and information propagation substep needs to be carried out, each with complexity of at most $N$ messages.

\section{B. Decreasing Power}

When the contours overlap, it will be required that the opportunistic transmitter decreases his transmission power. This however is relatively easy, since a node $N_{i}$ inside both contours will have an estimate of the received power from both transmitters, and can hence easily determine the $\Delta P_{i}$ causing the overlap in contours. A node noticing such an overlap simply communicates the $\Delta P_{i}$ to the second transmitter, that follows the largest power adjustment reported. This case is very unlikely to happen, but since we extrapolate an estimated pathloss model, we cannot avoid the possibility.

\section{RESUlTS}

In this section, we introduce the simulation setup, and discuss the main results.

\section{A. Simulation Model}

In order to verify the approach on a data set under known circumstances, we establish a simulation model. The simulation scene consists of a two-dimensional area in which buildings or obstacles result in shadowing losses $L_{S}$, on top of a general pathloss trend that varies with distance:

$$
L=10 \alpha \log _{10}(d)+\beta+L_{S}+X_{\sigma},
$$

where $L$ is the total pathloss in $d B(\alpha=4$ and $\beta=40 d B$ for the simulations). Since the measurement data shows a lot of variations in the measured RSSI, even for a fixed position $d$ and shadowing $L_{S}$, we also add zero-mean Gaussian distributed variations or noise $X_{\sigma}$. Following the ITU Terrain Model [11] $L_{S}$ is modeled as function of the depth of the line-of-sight ray into the building. The motivation behind this is that the wavefront can be modeled as a zone of energy
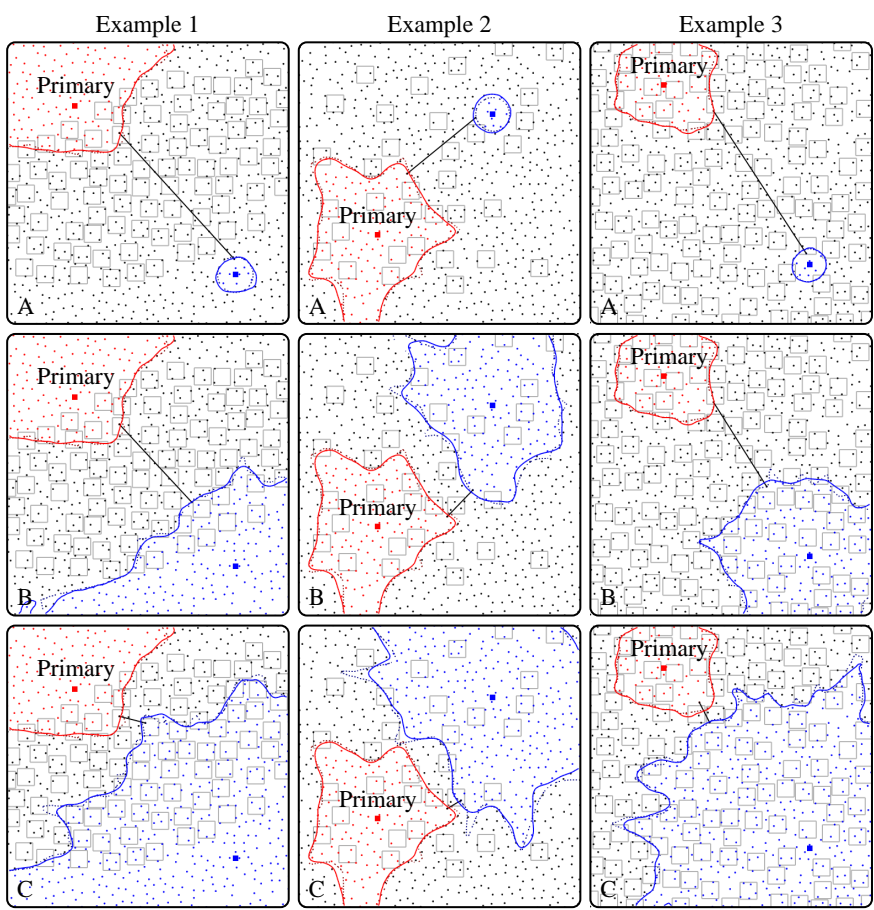

Fig. 6. Iterative power adjustment for three simulation scenarios of $1 \mathrm{~km}^{2}$ with communication range $h=95 \mathrm{~m}$, quadratic MLS and $\sigma_{N}^{2}=4$ which is the variance noticed on the outdoor measurements. Each box represents a building causing shadowing losses. The solid curves represent the approximation of the primary and secondary transmitter's interference contour. The dotted lines are the real (noise-free) contours. The straight line on each image corresponds to the contour-to-contour distance as computed by our algorithm. Row A shows for each example the resulting contour for the initial suboptimal power. Note the small area covered by the initial secondary contour. From the estimated local pathloss model at the secondary transmitter's contour point, a new transmission power is computed. Rows B and C show the result of this iterative process. Note the large difference between the secondary transmitter's coverage area at the original estimate in A and the resulting contour in C.

around the line-of-sight. Diffraction losses occur when part of this zone is obstructed. When the ray just passes next to the building, half of the zone is obstructed. When the ray passes in the middle of the building, the whole zone is obstructed, leading to larger losses. We however slightly adapt the model to allow received signals inside buildings. We note that the exact form of the model is not that important since our technique should work for any possible situation. The resulting shadowing loss is

$$
L_{S}=\max \left\{0,\left[5.125 \times\left(2-\frac{\left\|\mathbf{x}_{L}-\mathbf{x}_{O}\right\|}{D / 2}\right)\right]\right\},
$$

where $\mathbf{x}_{O}$ is the position of the obstacle, $D$ its width and $\mathbf{x}_{L}$ the orthogonal projection of $\mathbf{x}_{O}$ on the ray. The fraction $\frac{\left\|\mathbf{x}_{L}-\mathbf{x}_{O}\right\|}{D / 2}$ tells us how close to the center of the building the line-of-sight ray crosses. When the line-of-sight ray crosses the building through the center, we have a maximal $L_{S}$ of $10.25 d B$ per obstacle which is a value chosen from [3]. From the center of the building, the shadowing loss decreases linearly until $0 d B$.

\section{B. Results and Discussion}

The resulting iterative power adjustment is illustrated for three examples in each column of Fig. 6 . The primary and 
secondary senders' contour approximations are drawn using a solid line. The real contours are drawn as dashed lines. The computed minimal contour-to-contour distance is indicated by the straight line connecting the two corresponding nodes. The buildings are drawn as gray boxes. The simulation model as described above was used, with a noise power $\sigma_{N}=$ 4 corresponding to the real-world measurements. We used quadratic MLS with a kernel width of $95 \mathrm{~m}$ in all examples. Table I shows statistics for the three scenarios.

As can be seen in all images and Table I, the computed contours approximate the exact ones very well: Only $0.7 \%$ on average of all nodes was misclassified. Note that these 2D contour curves are in reality not constructed, but nodes locally make a binary classification as being interior or not. The curves are shown only for illustration purposes.

The images in row A show the secondary contour resulting from the initial power estimate based on the distance between the secondary sender and the primary contour. An optimistic pathloss model with $\alpha=2$ was used for the computation of the secondary transmitter's power as function of the measured distance from the first transmitter. Since actual propagation is by definition worse than the most optimistic condition, the coverage of the secondary transmitter is typically very small using this power, as can be seen in Fig. 6, A.

Our algorithm however iteratively estimates a more realistic pathloss model for the secondary transmitter, and as a result achieves a much more realistic power adjustment already after one iteration. As can be seen in Fig. 6, the resulting coverage of the secondary transmitter can be increased considerably in each of the three scenarios, without causing interference (i.e., overlap of the contours) to the first transmitter. Each power adjustment step (shown in B and C) requires estimation of the secondary transmitter's contour at the cost of $N$ transmissions. Next, each node inside the contour requires sending its distance to the first contour and its estimated pathloss, at the cost of $N_{\text {interior }} \leq N$ transmissions. As can be seen on the final images $\mathrm{C}$, a much larger area is covered by the secondary transmitter as compared to the initial configuration shown in A.

Our algorithm not only results in improved spatial reuse gain, but also relaxes the opportunistic transmitters' sensitivity requirements. Indeed, traditional approaches would require each possible transmitter to be able to sense the first transmitter. For a given target power of the second transmitter, this results in very high sensitivity requirements. In our approach, these requirements are relaxed, and the first transmitter's propagation contour is estimated close to the first transmitter and then efficiently propagated. Both this estimation and propagation are cheap and require only $N$ and a little over $N$ (as indicated in Table I) transmissions respectively. Typically, the algorithm converges in two to three iterations.

Due to the approximate inside/outside classification, we can not guarantee that the real final contours do not overlap and little interference might occur. As can be seen on the images, only in example 2 the final contours overlap, though for a negligible amount. However, it would be interesting future research to quantify the amount of possible overlap.
TABLE I

PERFormanCE OF THE ALGORITHM FOR THE EXAMPLES OF Fig. 6.

\begin{tabular}{cccc}
\hline & & $\begin{array}{c}\text { number of } \\
\text { updates }\end{array}$ & $\begin{array}{c}\text { percentage } \\
\text { misclassified }\end{array}$ \\
\hline \multirow{2}{*}{ example 1 } & primary sender & 724 & 0.28 \\
(710 nodes) & secondary sender & 721 & 0.14 \\
& secondary sender update 1 & 722 & 0.98 \\
& secondary sender update 2 & 714 & 1.12 \\
\hline \multirow{2}{*}{ example 2 } & primary sender & 732 & 0.83 \\
(723 nodes) & secondary sender & 730 & 0.14 \\
& secondary sender update 1 & 732 & 1.24 \\
& secondary sender update 2 & 732 & 2.21 \\
\hline \multirow{3}{*}{ example 3 } & primary sender & 721 & 0.70 \\
(712 nodes) & secondary sender & 723 & 0 \\
& secondary sender update 1 & 722 & 0.14 \\
& secondary sender update 2 & 719 & 0.98 \\
\hline
\end{tabular}

\section{CONCLUSION}

In this paper, we propose a new approach for iterative power control where to goal is to maximize spatial reuse while avoiding unwanted interference to existing networks. First, the propagation contours of the existing networks are estimated and efficiently propagated. Next, the secondary transmitter's power control is iteratively adapted based on estimates of real pathloss conditions. The main concepts behind the design of the scheme are motivated from outdoor measurements. The resulting scheme is shown to result in a much improved spatial reuse compared to traditional approaches.

\section{ACKNOWLEDGEMENTS}

The authors would like to thank Prof. Pravin Varaiya for his useful suggestions. Sofie Pollin is funded by a Marie Curie Outgoing International Fellowship of the EU. Bart Adams is funded as a post-doctoral researcher by the Fund for Scientific Research, Flanders (F.W.O.-Vlaanderen).

\section{REFERENCES}

[1] Q. Zhao and B.M. Sadler, A Survey of Dynamic Spectrum Access: Signal Processing, Networking, and Regulatory Policy, IEEE Signal Processing Magazine, vol. 24, no. 3, pp. 79-89, May, 2007.

[2] D. Mitra, An asynchronous distributed algorithm for power control in cellular radion systems, Proc. 4th WINLAB Workshop, Pct. 1993, pp. 249-257.

[3] A. Sahai, N. Hoven, S. M. Mishra, and R. Tandra, Fundamental tradeoffs in robust spectrum sensing for opportunistic frequency reuse, Tech Report, Mar 2006.

[4] C. Cordeiro, Challapali, Birru and S. Shankar, IEEE 802.22: An Introduction to the First Wireless Standard based on Cognitive Radios, Journal of Communications, Vol. 1, No. 1, April 2006.

[5] W. C. Y. Lee, Estimate of Local Average Power of a Mobile Radio Signal, IEEE Trans. on Vehicular Technology, Feb. 1985.

[6] K. Yonezawa, T. Maeyama, H. Iwai, H. Harada, Path Loss Measurement in $5 \mathrm{GHz}$ Macro Cellular Systems and Consideration of Extending Existing Path Loss Prediction Methods, WCNC 2004.

[7] H. Lim, C. Kim, Multicast tree construction and flooding in wireless ad hoc networks, Proceedings of the 3rd ACM international workshop on Modeling, analysis and simulation of wireless and mobile systems

[8] M. Mauve, J. Widmer, H. Hartenstein, A Survey on Position-Based Routing in Mobile Ad Hoc Networks, IEEE Network, Nov. 2001.

[9] F.Y. Chen, A. S. Lu, L. Zhang, A scalable solution to minimum cost forwarding in large sensornetworks, Computer Communications and Networks, 2001.

[10] B.D. Ripley, Statistical Inference for Spatial Processes, Cambridge University Press, 1988

[11] John S. Seybold, Introduction to RF propagation, 2005, Wiley. 